\title{
Modal analysis application for the problemof gamma- ray spectrometers resolution improving
}

\author{
Vladislav Malgin', Oḷegs Jakovḷevs² ${ }^{2}$ Jānis Vība ${ }^{3}$ \\ ${ }^{1,2}$ Baltic Scientific Instruments (BSI), Riga, Latvia \\ ${ }^{2,}{ }^{3}$ Riga Technical University (RTU), Riga, Latvia \\ ${ }^{2}$ Corresponding author \\ E-mail: ${ }^{1}$ v.malgin@bsi.lv, ${ }^{2}$ o.yakovlev@bsi.lv, ${ }^{3}$ janis.viba@rtu.lv
}

Received 28 August 2019; accepted 4 September 2019 DOI https://doi.org/10.21595/vp.2019.20974

Check for updates

Copyright (C) 2019 Vladislav Malgin, et al. This is an open access article distributed under the Creative Commons Attribution License, which permits unrestricted use, distribution, and reproduction in any medium, provided the original work is properly cited.

\begin{abstract}
The cryostat of gamma-ray spectrometer with HPGe detector cooled by Stirling cycle cryocooler is under consideration. The natural frequencies and modes shapes for components involved to oscillatory systems distinguished in dynamic model of cryostat were calculated by Solidworks simulation. The results obtained for the gamma-ray spectrometer "Monolith" produced by BSI, represent its specific modal portrait and allow identification the components of electrical noise due to microphone effect. The absence of resonant amplification due to coincidence between the harmonics of cryocooler operating frequency and the natural frequencies of the cryostat components is an important condition for reducing the influence of microphonics. The results obtained by modal analysis are also useful for assessing the influence of external mechanical disturbances, such as shocks, external vibration or audible noise.
\end{abstract}

Keywords: gamma-ray spectrometers, microphonic noise, modal analysis.

\section{Introduction}

Spectrometers based on High Purity Germanium (HPGe) detectors are widely used for radiation monitoring. Radioisotope identification is based on measuring the energy of emitted gamma rays. In the presence of gamma radiation, the detector generates a current pulse with an amplitude proportional to the energy of gamma quantum. Further, after amplifying by a low noise preamplifier and shaping these current pulses are fed to a multichannel amplitude analyzer for sorting. Particle identification through pulse shape discrimination is used mainly in nuclear physics.

The presence of noise in the signal path increases the error in measuring the current pulse amplitude and reduces the resolution of a spectrometer. The applying of HPGe detectors in a cryostat of a gamma-ray spectrometers assumes their cooling to cryogenic temperatures for low noise performance. Cooling by means of electromechanical Stirling cryocooler without Dewar with a liquid nitrogen is very convenient for users. However, mechanical vibrations inherent for cryocoolers are the cause of electrical noise induced at the preamplifier input by microphone effect (the conversion of mechanical vibrations into electrical signals). This fact reduces the final resolution of spectrometer. Usually, to reduce the influence of microphone effect, the electronic methods are used. There are: the applying of band pass filters in input circuits of preamplifier together with selection of the shortest shaping time or input circuits with two shaping times. In some case the digital filters in subsequent blocks of spectrometric signal processing are applied [1-3]. To further reducing of microphone effect, more complicated for practical implementation methods of active vibration cancellation are used [4-6].

Note that the approaches mentioned above, are quite effective, but are based at reducing the result of microphone effect, but not its cause. As a rule, the vibration force produced by the used types of cryocoolers is not big and does not exceed 5-10 N. The most significant for the appearance of microphone effect is the probable resonant amplification of vibrations generated by cryocooler in the oscillatory systems formed by the components of spectrometer. In this case, the resolution of the spectrometer can degrade significantly. The aim of this investigation is to evaluate the 
resonant frequencies of cryostat, which can significantly affect to the microphone effect in the gamma-ray spectrometer "Monolith" produced by BSI, Fig. 1(a).

In the following Sections, the essence of the approach for identifying noise signals caused by the microphone effect, as well as the results of modal analysis of the cryostat are presented.

\section{Search for microphonic components in the detector signal}

To provide maximum resolution of the gamma-ray spectrometer during it tuning is necessary to identify the source of the noise components and minimize it. Electric noise in a signal path is a sum of electromagnetic interference and the signals from microphonics. The microphone components of the noise are most significant when the resonant amplification of vibrations in components of the cryostat occurs. Note, that the direct installation of accelerometers for vibration measuring in a vacuum chamber of a cryostat with HPGe detector is usually not possible. Therefore, the proposed approach based on a modal analysis of the cryostat components may be useful.

The spectrum analyzer allows obtain the noise spectrum in the low frequency domain, Fig. 2 . Comparison of the peak frequencies obtained by measurement with the results of a modal analysis makes it possible to diagnose the nature of main noise components and its possible source. Identification of microphone noise components helps to find the useful decisions for elimination or reducing them. The sequence of actions proposed is presented in Fig. 1(b).

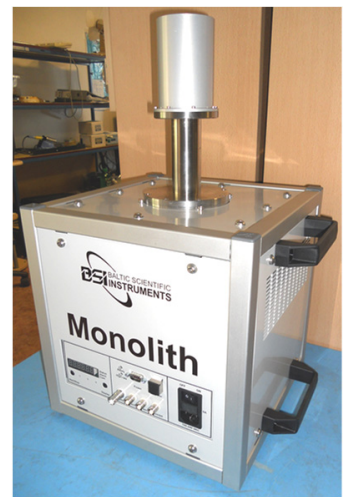

a)

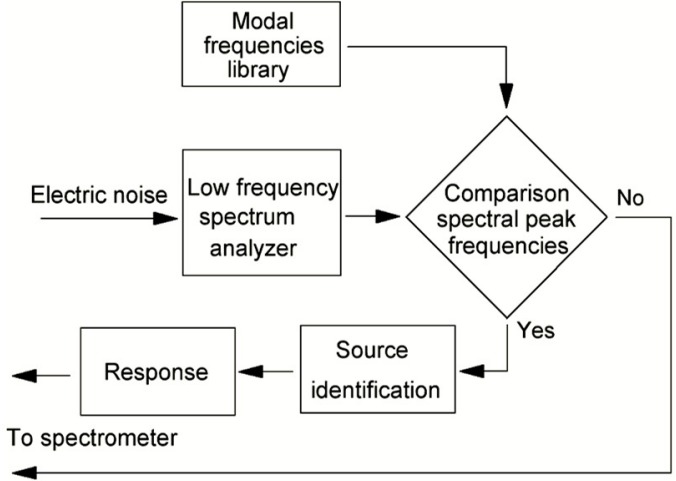

b)

Fig. 1. Gamma-ray spectrometer "Monolith" produced by BSI and analysis of microphonic noise

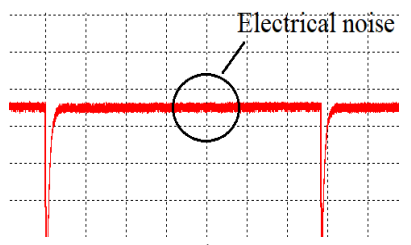

a)

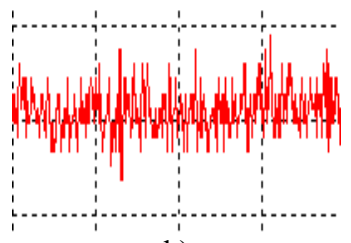

b)

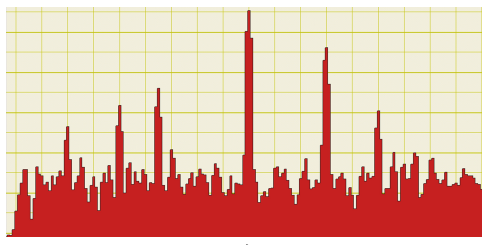

c)

Fig. 2. The electrical signals on output of preamplifier: a) current pulses; b) electrical noise; c) spectrum of noise in low frequency domain

\section{The dynamic model of cryostat with HPGe detector}

A typical design of a cryostat with Stirling cryocooler is shown in Fig. 3. The four oscillatory systems, whose mechanical vibrations cause a microphone effect can be distinguished:

- the first system is formed by a massive cryostat with cryocooler mounted on an elastic plate or rubber studs;

- the second system is formed by a HPGe crystal placed into aluminum holder and mounted 
on rigid base of cryostat by supports having a certain flexibility;

- the third and fourth systems are formed by a thin-walled vacuum cap and thermal shield.

Modern equipment design involves the use of computer software packages in which modal analysis of components is included as an option. This is convenient for practical calculations using the Finite Element Method, since all components have a complex shape. The design of gamma spectrometer "Monolith" in BSI was performed in the Solidworks environment. The lowest modes of oscillatory systems mentioned above are determined by Solidworks simulation and presented in Figs. 4-7.

The gamma- ray spectrometer "Monolith" can be equipped with HPGe crystals of various size and mass, which are determined by the required efficiency [7]. The mass of the installed crystal can vary from 0.5 to $1.8 \mathrm{~kg}$ or more. The frequencies of the lowest modes for a detector with a relative efficiency of $15 \%$ and a mass of $0.78 \mathrm{~kg}$ are presented in Fig. 5. When HPGe crystals with greater efficiency (and mass, respectively) are used the mode frequencies decrease.

The operating frequency of Stirling coolers used in "Monolith" lies in the range from $60 \mathrm{~Hz}$ to $100 \mathrm{~Hz}$. A significant vibration reduction at the driving frequency is usually provided by a tuned vibration absorber. However, its harmonics can cause resonant oscillations of oscillatory systems [7].

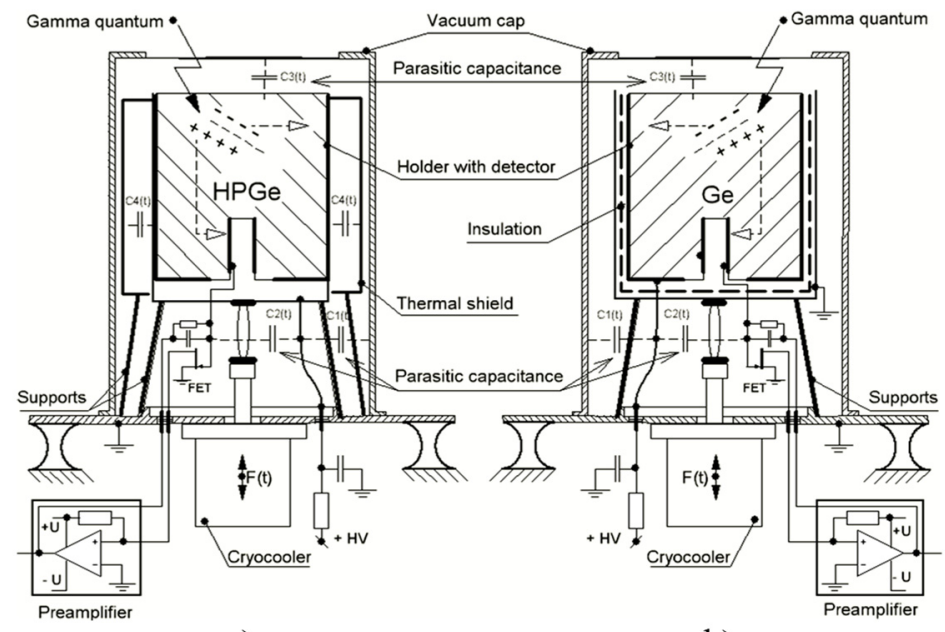

a)

b)

Fig. 3. Typical design of cryostat: a) with non-insulated detector; b) with insulated detector

The output capacitance of HPGe detector is very small and does not exceed a few $\mathrm{pF}$. Therefore, even minor changes in parasitic capacitances due to mechanical vibration, lead to a periodic change in charge at the input of preamplifier. Note that the microphone effect appears only in the presence of a large potential difference between the vibrating surfaces. So, in a cryostat with a non-insulated detector, Fig. 3(a), the microphone effect is affected by the capacitance between the detector holder and the vacuum cap or thermal shield if it is installed. In contrary, in a cryostat with an insulated detector, Fig. 3(b), the main source of microphone effect is the capacitance between the detector and the input window of vacuum cap. Cryostat vibrations can also cause high voltage wires to vibrate.

Not all oscillation modes cause the appearance of a significant microphone effect. For example, the detector's oscillations in the torsional mode have practically no effect on the parasitic capacitances. Moreover, it is important to take into account the Q-factor of considered oscillatory systems, which is determined by the energy dissipation in the material and elastic supports. For example, the Q-factor of the detector assembly with fiberglass supports for the axial vibration mode does not exceed 10-15 [7]. The Q-factor of the axial resonances of the vacuum cap is much higher and reaches values of 50-70. 


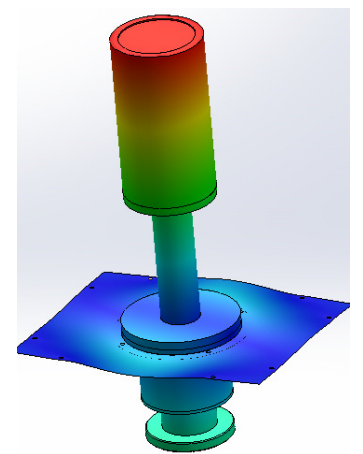

a) $41 \mathrm{~Hz}$

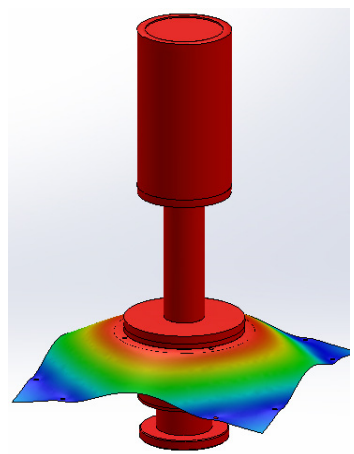

b) $67 \mathrm{~Hz}$

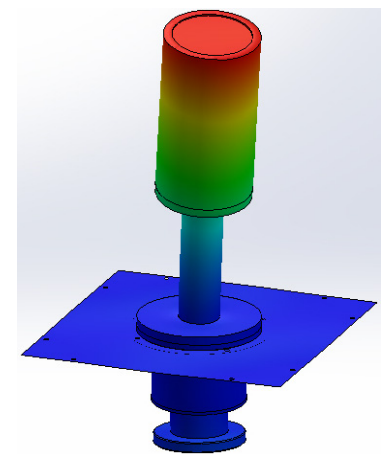

c) $298 \mathrm{~Hz}$

Fig. 4. The lowest modes of cryostat assembly: a), c) bending; b) axial

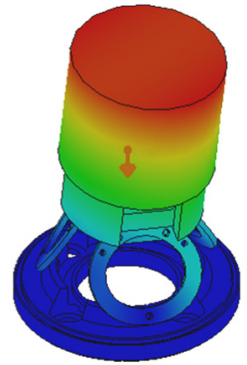

a) $120 \mathrm{~Hz}$

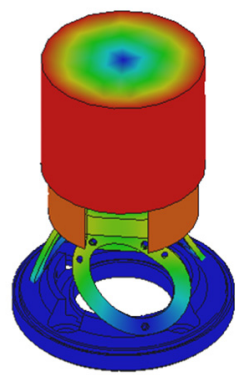

b) $406 \mathrm{~Hz}$

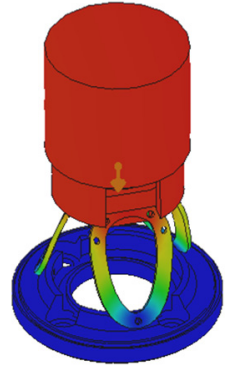

c) $454 \mathrm{~Hz}$

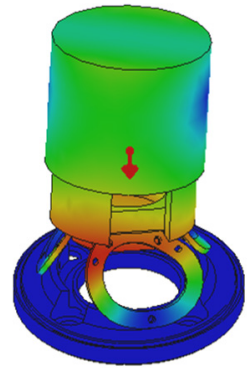

d) $679 \mathrm{~Hz}$

Fig. 5. The lowest modes of detector's assembly: a), d) bending; b) torsion; c) axial

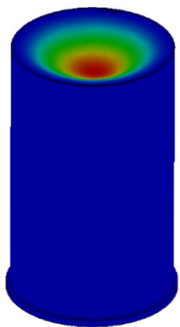

a) $1049 \mathrm{~Hz}$

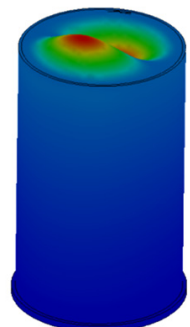

b) $2273 \mathrm{~Hz}$

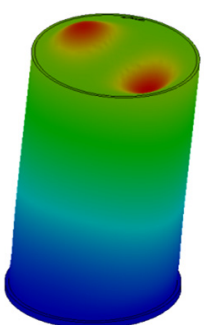

c) $4110 \mathrm{~Hz}$

Fig. 6. The lowest modes of vacuum cap: a), b) axial; c) bending

\section{The modal analysis of thermal shield}

The cooling of large size germanium detectors up to cryogenic temperatures by application of low-power cryocoolers results in the topical task of the thermal losses' decreasing. An effective method to reduce heat transfer in cryostat due to thermal radiation and molecular conductivity of residual gases is the application of thermal shields. Structurally, thermal shields are thin-walled cylinders fixed on the plastic supports coaxially between the cooled detector and the vacuum cap, Fig. 3(a). For example, the thermal shield for "Monolith" has outer diameter $72 \mathrm{~mm}$, height $100 \mathrm{~mm}$, wall thickness $0.5 \mathrm{~mm}$ and is made from a stainless steel. The lowest modes are determined by Solidworks simulation and presented in Fig. 7.

In presence of a heat shield located coaxially between the non-insulated detector and the vacuum cap, Fig. 3, a, the values of parasitic capacitances and as a result, the microphone effect increase. To reduce this effect, a thermal shield should be made with a shell thickness that will ensure the absence of possible resonance at the harmonics of the cryocooler's operating frequency. 
The dependence of the resonant frequencies of the thermal shield on the shell thickness is presented in Fig. 8. The axial and torsional modes do not change the value of parasitic capacitances and do not affect the microphonic.

As was mentioned above the microphone effect arises between oscillatory systems with a large voltage (potential) difference only. Therefore, in cryostats with a non-isolated detector, Fig. 3(a), the shell of the thermal shield must also be connected to high voltage as well as the detector. This avoids the microphone effect due to detector vibrations.

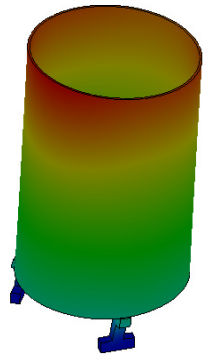

a) $219 \mathrm{~Hz}$

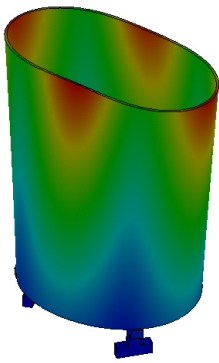

b) $310 \mathrm{~Hz}$

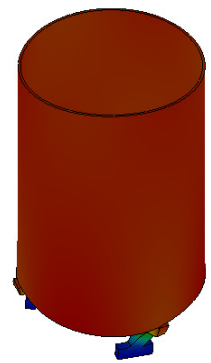

c) $348 \mathrm{~Hz}$

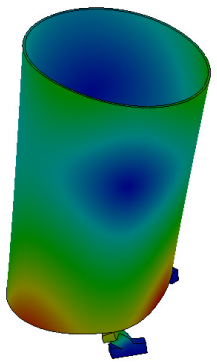

d) $796 \mathrm{~Hz}$

Fig. 7. The lowest modes of thermal shield made from stainless steel, thickness $0.5 \mathrm{~mm}$ : a) bending; c) torsion; b), d) shell

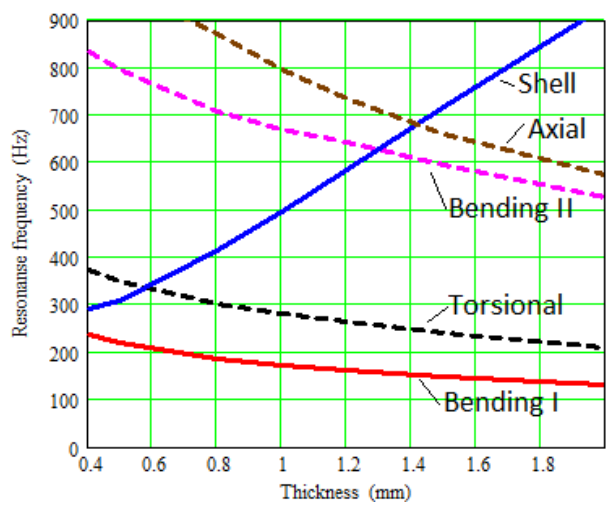

Fig. 8. Dependence of the resonant frequencies of the thermal shield on the shell thickness

\section{Conclusions}

Modal analysis based on the compiled dynamic model of cryostat for gamma-ray spectrometer "Monolith" was carried out. The modes of cryostat components calculated by Solidworks simulation made it possible to determine the most probable domains for resonant amplification of vibration generated by the cryocooler. The procedure presented in Section 2 allows identification the components of electrical noise due to microphonics. In case of resonant amplification at the operating frequency of cryocooler or its harmonics, the controller allows to change the value of operating frequency. The absence of coincidence between the frequencies of cryocooler vibrations and the natural frequencies of the cryostat components is an important condition for reducing the influence of microphonics.

Note, the wide range of HPGe detectors with various mass-dimensional characteristics are used in gamma-ray spectrometer. The resonant frequencies calculated for a cryostat with a given detector represent its specific modal portrait, Table 1 . In practice, similar calculations should be performed for cryostats with each type of HPGe detector.

The results obtained by modal analysis are also useful for assessing the influence of external mechanical disturbances, such as shocks, external vibration or audible noise. 
The results are used in Baltic Scientific Instruments company for individual tuning of gamma-ray spectrometers" Monolith" during the production process to achieve the best resolution.

Table 1. The frequencies of lowest modes for cryostat with HPGe detector with $15 \%$ efficiency

\begin{tabular}{|c|c|c|c|c|c|}
\hline & \multicolumn{4}{|c|}{ Frequencies of presented modes } & \\
\hline Spectrometer's unit & Bending I & Torsion & Bending II & Axial & Shell \\
\hline Cryostat & $41 \mathrm{~Hz}$ & - & $298 \mathrm{~Hz}$ & $67 \mathrm{~Hz}$ & - \\
\hline Detector $15 \%$ on supports & $120 \mathrm{~Hz}$ & $406 \mathrm{~Hz}$ & $679 \mathrm{~Hz}$ & $454 \mathrm{~Hz}$ & - \\
\hline Thermal shield $(0.5 \mathrm{~mm})$ & $219 \mathrm{~Hz}$ & $348 \mathrm{~Hz}$ & - & $796 \mathrm{~Hz}$ & $310 \mathrm{~Hz}$ \\
\hline Vacuum cap & $4110 \mathrm{~Hz}$ & - & - & $1049 \mathrm{~Hz}$ & - \\
\hline
\end{tabular}

\section{References}

[1] Schultz M. K., Keyser R. M., Trammell R. C., et al. Improvement of spectral resolution in the presence of periodic noise and microphonics for hyper-pure germanium detector gamma-ray spectrometry using a new digital filter. Journal of Radioanalytical and Nuclear Chemistry, Vol. 271, Issue 1, 2007, p. 101-106.

[2] Barton P., Amman M., Martin, Vetter R. K. Ultra-low noise mechanically cooled germanium detector. Nuclear Instruments and Methods in Physics Research Section A: Accelerators, Spectrometers, Detectors and Associated Equipment, Vol. 812, 2016, p. 17-23.

[3] Keyser R. M., Bingham R. D., Twomey T. R. Improved performance in germanium detector gamma-spectrometers based on signal processing. Journal of Radioanalytical and Nuclear Chemistry, Vol. 276, Issue 3, 2008, p. 567-575.

[4] Hassan A., Torres Perez A., Kaczmarczyk S., Picton P. Vibration control of a Stirling engine with an electromagnetic active tuned mass damper. Control Engineering Practice, Vol. 51, 2016, p. 108-120.

[5] Johnson W., Long R., Nelson M., Mascarenas D. Embedded active vibration cancelation of a piston - driven cryocooler for nuclear spectrometry applications. Proceedings of the International Modal Analysis Conference, California, 2012, p. 377-384.

[6] Zimmermann S. Active microphonic noise cancellation in radiation detectors. Nuclear Instruments and Methods in Physics Research Section A: Accelerators, Spectrometers, Detectors and Associated Equipment, Vol. 729, 2013, p. 404-409.

[7] Jakovlevs O., Malgin V., Vība J. Modal analysis of HPGe detector assembly in gamma-ray spectrometers. Journal of Vibroengineering, Vol. 16, 2017, p. 61-66. 